\title{
Die Arzthaftung von gestern und das Medizinrecht von heute in rechtsvergleichender Perspektive
}

\author{
Amalia Diurni
}

\section{Die Geschichte der Arzthaftung}

Es ist unmöglich, eine historisch-rechtsvergleichende Abhandlung zum Medizinrecht zu verfassen, ohne von Erwin Deutsch zu sprechen; ebenso unmöglich ist es, einen Beitrag zur Festschrift für Erwin Deutsch zu schreiben, ohne mit der Geschichte der Medizin zu beginnen.

\section{Vom römischen Recht bis zum 19. Jahrhundert}

Nach Auffassung der Lehre ${ }^{1}$ stellte das Arzt-Patienten-Verhältnis im römischen Recht keine auf die beiden Elemente der Dienstleistung und der Zahlung einer Vergütung gestützte locatio-conductio dar, denn ein solches Verhältnis wurde als erniedrigend empfunden und war vorzugsweise den Sklaven bzw. Freigelassenen vorbehalten. Auch die Unterscheidung zwischen locatio operis und locatio operarum $^{2}$ war für die Arzt-Patienten-Beziehung nicht von Bedeutung. Arzt und Patient waren - ebenso wie Anwalt und Mandant - nicht rechtlich aneinander gebunden. Der Austausch zwischen der Leistung und der eventuellen Prämie (im Erfolgsfälle) ${ }^{3}$ war durch den mos geregelt. Der honos stellte das Geschenk dar, das einer Person als Zeichen der Schätzung überreicht wurde, und aus dem Adjektiv honorarius entstand das Substantiv honorarium, das bereits im 2. Jahrhundert $\mathrm{n}$. Chr.

1 PESCANI, voce Onorari (diritto romano), in Noviss. dig. it., Bd. XI, Torino, 1965, S. 929.

2 VOLTERRA, Istituzioni di diritto privato romano, Roma, 1980, S. 515 f.; A. MASI, voce Locazione in generale (Storia), in Enc. dir., Bd. XXIV, Milano, 1974, S. 908 ff.; INZITARI, Autonomia privata e controllo pubblico nel rapporto di locazione, Napoli, 1979, S. 5; RESCIGNO, voce Obbligazioni (nozioni), in Enc. dir., Bd. XXIX, Milano, 1979, S. $190 \mathrm{f}$.

3 PEZZANO, voce Onorario, in Enc. dir., Bd. XXX, Milano, 1980, S. 175 ff. 
in der Fachsprache die Vergütung des „professionista” (Angehöriger einer Berufskategorie) bezeichnete.

Der Arzt konnte die Vergütung nicht einklagen, ihm wurde - wenn überhaupt - nur eine actio extra ordinem zuerkannt ${ }^{4}$. Nach dem Codex Theodosianus aus dem Jahr 428 n. Chr. war unter dem Titel De professoribus et medicis ${ }^{5}$ eine Vergütung nur im Falle einer erfolgreichen Behandlung zulässig. Die Forderung von Vorauszahlungen war dem Arzt dagegen untersagt. Auch die Digesten ${ }^{6}$ enthalten Bestimmungen, die verhindern sollen, dass der Arzt seine dem Kranken überlegene Stellung ausnutzt.

Die genaueste Regelung der ,,patti di guarigione“ (Heilungsverträge) der Spätantike und des Hochmittelalters befindet sich in den Leges Visigothorum ${ }^{7}$, wonach

4 Vgl. BOZZONI, I medici e il diritto romano, Napoli, 1904, S. 200 ff. Die Quelle der actio extra ordinem der Ärzte infolge der mangelnden Zahlung der Vergütung findet sich in den Digesten 50, 13: De variis et extraordinariis cognitionibus. Nach dem Gesetz Cincia (Lucio Cincio Alimento) de donis et muneribus (204 v. Chr.), übernommen von Tacito [Tac., Ann., 11, 5 (anno 47 d.C.)], war die Entgegennahme einer Vergütung vor der Erbringung der Leistung verboten.

5 Codex Theodosianus, XIII, 3, 8: Impp. Valentinianus et Valens A.A. ad Praetextatum P. $\mathrm{V}$ : „Exceptis portus Syxti virginumque vestalium, quot regiones urbi sunt, totidem consistuantur archiatri, qui scientes annonaria sibi commoda a populi commodis honeste obsequi tenuioribus malint, quam turpiter servire divitibus. Quos etiam ea patimur accipere, quae sani offerunt pro obsequiis, non ea quae periclitantes pro salute promittunt [...]". Dieser Abschnitt wurde im Codex Iustinianus, 10, 53, De professoribus et medicis, 9, zur Gänze wiedergegeben und war Gegenstand von Anmerkungen und Kommentaren. Vgl. NUTTON, Archiatri and the Medical Profession in Antiquity, in Papers of the British School in Rome, Bd. XLV, 1977, S. 191 ff.; BELOW, Der Arzt im römischen Recht, München, 1953.

6 Digesten, 50, 13, 3, Si medicus (Ulpiano, Libro octavo de omnibus tribunalibus, III sec.): „Si medicus, cui curandos suos oculos, qui eis laborabat, commiserat, periculum amittendorum eorum per adversa medicamenta inferendo compulit, ut ei possessiones suas contra fidem bonam aeger venderet: incivile factum praeses provinciae coerceat remque restitui iubeat".

7 Leges Wisigothorum, Dei medici e dei malati, in Leges Wisigothorum Antiquiores, Aufl. Zeumer, Hannover-Leipzig, 1894, Lib. XI, Tit. I, S. 292: „Lex III. Antiqua <Si medicus pro aegritudine ad placitum expectetur: Si quis medicum ad placitum pro infirmo visitando aut vilnere curando poposcerit: ut viderit vulnus medicus aut dolores agnoverit, statim sub certo placito cautione emissa infirmum suscipiat>”; „Lex IV. Antiqua < $\mathrm{Si}$ ad placitum susceptus moriatur infirmus: Si quis medicus infirmum ad placitum susceperit, cautionis emisso vinculo infirmum restituat sanitati; certe si periculum contigerit mortis, mercedem placiti penitus non requirat; nec ulla inde utrique parti calumnia moveatur $>$ ”; „Lex V. Antiqua $<\mathrm{Si}$ de oculis medicus hypochymata tollat: Si quis medicus hypochyma de oculis abstulerit et ad pristinam sanitatem perduxerit infirmum, V. solidos pro suo beneficio consequatur $>$ ". Vgl. GARRISON, An Introduction of the History of Medicine, Philadelphia, 1929, S. 146; NEUBURGER, History of Medicine, Bd. II, Oxford, 1925, S. 10 f.; AMUNDSEN, Visigothic Medical Legislation, in Bull. Hist. Med., Bd. XLV, n. 6, 1971, S. 553 ff. 
Die Arzthaftung von gestern und das Medizinrecht von heute in rechtsvergleichender Perspektive

das Arzt-Patient-Verhältnis durch eine Vereinbarung entsteht. Die typische Verpflichtung besteht in einem Leistungserfolg: Der Arzt muss den Kranken heilen; nur die erfolgreiche Behandlung berechtigt zur Zahlung der Vergütung: „Falls der Kranke verstirbt, kann der Arzt die vertraglich vereinbarte Prämie selbstverständlich nicht verlangen"8.

Diese Bestimmungen bildeten die Rechtsgrundlage der im Mittelalter üblichen „Heilungsverträge“, die vor einem Notar geschlossen wurden und als „öffentliche Urkunden" anerkannt waren'. In diesen Verträgen wurden die Vergütung, die Frist und die Methoden der Heilung festgelegt. ${ }^{10}$ Die medizinische Praxis war also im Grenzbereich zwischen den Handelsbeziehungen und der gegenseitigen Unterstützung angesiedelt: Gegenstand des Erwerbs war nicht die konkrete Leistung oder ein bestimmtes Medikament, sondern das Ergebnis der Behandlung bzw. die Heilung. Die eigentliche therapeutische Beziehung entsprang der gegenseitigen Solidarität, die auf die römische Tradition zurückgeht ${ }^{11}$ und dann in die christliche Ideologie übernommen wurde ${ }^{12}$.

Im ancien régime genoss der Patient also besondere, anerkannte Privilegien ${ }^{13}$, und ihm war das Urteil über die Tätigkeit des Arztes überlassen. Der diesbezügliche Wandel beginnt ab der ersten Hälfte des 17. Jahrhunderts, als die Haftung des Arztes nicht mehr unter dem Gesichtspunkt der Heilung bewertet wird, sondern je nachdem, ob die angewendeten Therapien und Medikamente den traditionellen Behandlungen entsprechen, ${ }^{14}$ und sich der Grundsatz durchsetzt, dass die berufliche Tätigkeit, die der Arzt - kraft eines öffentlich verliehenen Titels - als rechtmäßiges Mitglied der entsprechenden Kategorie ausübt, nach Zeitaufwand und Leistung zu bezahlen ist. Dadurch kommt es zu einer Professionalisierung des

$8 \quad$ Vgl. Lex IV, Antiqua, a.a.O.

9 POMATA, La promessa di guarigione - Malati e curatori in antico regime, Bari, 1994, S. 94, dessen Anhang verschiedene Beispiele für Verträge und Urkunden mit Heilungsversprechen von 1244 bis 1764 enthält.

10 Vgl. PILLIO MEDICINENSE, Quaestiones Sabbatinae, Augustae Taurinorum, 1967, C.G.J.C. IV, Quaestio XII, S. 20 f.; BARTOLO DA SASSOFERRATO, In secundam Digesti veteris partem, Venezia, 1570, II, 136, in den Anmerkung zu den Digesten 19, 2, 59, 1. „Marcius“. Unter den Kanonisten, DURANDUS, Speculum Judiciale, Bononiae, 1477 [1271], „de salariis“, 201; ANGELUS DE CLAVASIO, Summa, Venezia, 1487, „medicus“, Rn. 14, 235; vgl. unter den Zivilrechtlern TIRAQUEAU, Commentariorum in 1. Si unquam. C. de revoc. don., VI, Frankfurt, 1574 (1535), S. 237, Rn. 180; BERTACHINI, Repertorium iuris utriusque Doctoris praestantissimi, Venezia, 1570, „medicus“, 332-334.

11 Vgl. PESCANI, a.a.O., S. 929.

12 Die Idealvorstellung des Arztes, der seine Kunst unentgeltlich zur Verfügung stellt, geht auf die Heiligen Kosmas und Damian zurück und ist im Mittelalter stark in der Volkskultur verwurzelt (s. WITTMANN, Kosmas und Damian: Kultausbreitung und Volksdevotion, Berlin, 1967, S. 12 ff.). Auch mit dem Heiligen werden Vereinbarungen getroffen, und auch er wird um Heilung gebeten, wie die ex-voto bezeugen. Vgl. in diesem Zusammenhang BROWN, Il culto dei santi, trad. it. Torino, 1983, S. $162 \mathrm{ff}$.

13 TH. ACTIUS, De infirmitate eiusque privilegiis et affectibus, Venezia, 1603.

14 Vgl. POMATA, a.a.O., S. 289 ff. 
Arztberufes, und das Verstreichen der Zeit begünstigt nun nicht mehr den Patienten, sondern den Arzt. Die Beurteilung der ärztlichen Sorgfalt wird zu einer technischen Beurteilung, die nicht mehr Aufgabe des Patienten, sondern der zuständigen Behörde ist.

Ein weiterer, fundamentaler Schritt in der Geschichte der Medizin erfolgt im 19. Jahrhundert: Die auf die subjektive Symptomatologie des Patienten gestützte Konzeptualisierung wird durch eine Vorstellung abgelöst, in der die Krankheit zu einer individuellen und konkreten Identität wird: Der Patient ist einzig und allein der Träger dieser Krankheit. ${ }^{15}$ Das spekulative und auf die Analyse der Symptome aufgebaute ärztliche Wissen des ancien régime verwandelt sich in eine auf das Studium der Krankheit und der Therapie gestützte Wissenschaft.

In der Zwischenzeit setzen sich auch die Juristen mit dem Heilungsvertrag auseinander und erklären ihn schließlich der sozialen Entwicklung und den Wandlungen der Arztkategorie entsprechend ${ }^{16}$ für ungültig. Der Ansatz hierfür stammt aus einer Passage der Digesten ${ }^{17}$, wonach der Vertrag zwischen Anwalt und Mandant nur dann gültig ist, wenn sein Abschluss nicht vor oder während des Prozesses, sondern erst nach dessen Beendigung erfolgt ist. ${ }^{18}$ Dadurch wird der Gleichstellung zwischen Arzt und Patient ein Ende gesetzt.

Durch die Kombination aus wissenschaftlichem Fortschritt, beruflichem und sozialem Bewusstsein, wirtschaftlichem Interesse und juristischer Entwicklung entstand im 19. Jahrhundert das von den Soziologen als „,berufliche Dominanz“

15 Vgl. COHEN, The Evolution of the Concept of Disease, in LUSH (Hrsg.), Concepts of Medicine, Oxford, 1961.

16 Zur geschlichtlichen Entwicklung hinsichtlich anderer Berufsgruppen, vgl. PISCIONE, voce Professioni (disciplina delle), in Enc. dir., Bd. XXXVI, Milano, 1987, S. 1040 f.

17 Digesten, 50, 13, Si cui: „Si cui cautum est honorarium, vel si quis de lite pactus est: videamus an petere possit. Et quidem de pactis ita est rescriptum ab imperatore nostro, et divo patre ejus: Litis causae malo more pecuniam tibi promissam ipse quoque profiteris. Sed hoc ita jus est, si suspensa lite societatem futuri emolumenti cautio pollicetur. Si vero post causam actam est honoraria summa, peti poterit usque ad probabilem quantitatem, etsi nomine palmarii cautum sit: sic tamen, ut computetur id quod datum est, cum eo quod debetur, neutrumque compositum licitam quantitatem excedat". So auch im Justinianischen Kodex, II, 6, De postulando, 2: „Praeterea nullum cum eo litigatore contractum, quem in propriam recepit fidem, ineat advocatus: nullam conferat pactionem".

18 Die Kommentatoren und Juristen waren der Auffassung, dass der Lohn des Rechtsanwalts nach Beendigung der Leistung, d.h. nach dem Abschluss des Prozesses, zu entrichten war. Vgl. BARTOLO, In tres codicis libros, 242, gefolgt von JANOS MAYNUS, In primam Digesti veteris partem commentarii, Lugduni, 1569, 56: „Advocati non debent habere salarium lite durante [...] quod non debent habere salarium nisi finita causa“. BALDUS, In IV et V Codicis Libros Commentaria, 11, hat dagegen die moderne Vorstellung vorweggenommen und den Standpunkt vertreten, dass die Zahlung der Vergütung nach Beendigung des Prozesses den Klienten begünstige und dessen Nichterfüllung ermögliche. 
Die Arzthaftung von gestern und das Medizinrecht von heute in rechtsvergleichender Perspektive

bezeichnete Phänomen. ${ }^{19}$ Kraft dieser Dominanz neigen die Juristen erneut dazu, den Einsatz des vertraglichen Instruments in den Beziehungen zwischen Arzt und Patient sowie zwischen Anwalt und Mandant als rechtmäßig anzusehen. Der Heilungsvertrag gehört aber bereits der Vergangenheit an, denn die Zufallsbedingtheit und die Ungewissheit des Behandlungserfolgs bilden den empirischen Grund dafür, dass die Abhängigkeit zwischen Vergütung und Heilung unangemessen ist. Der Erfolg ist demnach autonom unter Berücksichtigung der bei der Leistungserbringung angewandten Sorgfalt zu beurteilen und kann nicht in obligatione abgeleitet werden. Als Gegenstand des Interesses des Patienten, das der Arzt zu befriedigen versucht - wenngleich er dazu nicht verpflichtet ist - wird der Erfolg nicht zu einem Bestandteil des Rechtsverhältnisses. Die berufliche Dominanz der Wissenschaft und der Therapien fördern gemeinsam mit dem Prinzip der sorgfältigen Leistung das Entstehen des Konzepts des fachlichen Ermessens ${ }^{20}$, und zwischen dem Klienten und dem professionista, der einem intellektuellen Beruf nachgeht, kommt ein Vertrag zustande, der für den Klienten als eine Art einseitiger aleatorischer Vertrag anzusehen is $\mathrm{t}^{21}$.

\section{Die Differenzierung zwischen Leistungshandlung und Leistungserfolg im französischen und italienischen Recht}

In diesem Zusammenhang hat die französische Lehre die Unterscheidung zwischen obligation de moyen( $(s)$ und obligation de résultat ${ }^{22}$ ausgearbeitet, die auf

19 FREIDSON, Professional Dominance, New York, 1970; STARR, The Social Transformation of American Medicine, New York, 1982.

20 D. CARUSI, Responsabilità del medico e obbligazione di mezzi, in Rass. dir. civ., 1991, S. 491; SANTORO PASSARELLI, Voce Professioni intellettuali, in Noviss. dig. it., Bd. XIV, Torino, 1957, S. 23 ff.; G. GIACOBBE, voce Professioni intellettuali, in Enc. dir., Bd. XXXVI, Milano, 1987, S. 1065 ff.

21 FORTINO, La responsabilità civile del professionista, Milano, 1984, S. 60; FRASCA, Verso un nuovo orientamento giurisprudenziale in tema di ripartizione degli oneri probatori nel caso di responsabilità contrattuale del medico, in La responsabilità medica, Milano, 1982, S. 148; PERULLI, Il lavoro autonomo, in Trattato di diritto civile e commerciale früher unter der Leitung von Cicu und Messineo, jetzt unter der Leitung von Mengoni, Bd. XXVII, T. 1, Milano, 1996, S. 448.

$22 \mathrm{Zu}$ den angeblich römischen Quellen der Unterscheidung vgl. MAZEAUD/TUNC, Traité théorique et pratique de la responsabilité civile délictuelle et contractuelle, Bd. I, 6. Aufl., Paris, 1965, S. 38; MAZEAUD, Essai de classification des obligations: obligations contractuelles et extracontractuelles, „obligations déterminées“ et „obligations générales de prudence et de diligence“, in Riv. trim. dr. civ., 1936, S. 27; vgl. MENGONI, Obbligazioni „di risultato“ e obbligazioni di „mezzi“, in Riv. dir. comm., 1954, I, S. 199 ff.; VISINTINI, Trattato breve della responsabilità civile, 3. Aufl., Padova, 2005, S. 90 ff. CANNATA, Le obbligazioni in generale, in Trattato dir. priv. unter der Leitung von Rescigno, Bd. 9, Obbligazioni e contratti, Torino, 1984, S. 36, verweist auf PAPINIANO, D. 22, 1, 4pr. Gegenteiliger Auffassung ist PERULLI, a.a.O., S. 426. 
Demogue zurückgeht ${ }^{23}$. Ihr Einfluss auf die Rechtsprechung war so stark ${ }^{24}$, dass die Fundamente der französischen Lehre regelrecht erschüttert wurden ${ }^{25}$.

Unter dem normativen Gesichtspunkt vertritt ein Teil der Lehre ${ }^{26}$ die Auffassung, dass sich Art. 1137 des französischen Code civil auf die Sorgfaltsregel bezieht und die Erfüllung der Leistungshandlungen charakterisiert, während in den Art. 1147 und 1148 fr. Cod. Civ. die Haftung für Nichterfüllung mangels Erreichung des Leistungserfolgs geregelt ist. Das italienische Rechtssystem folgt scheinbar demselben Ansatz, denn Art. 1176 des italienischen Codice civile betrifft die Leistungshandlung ${ }^{27}$ und Art. 1218 it. Cod. Civ. den Leistungserfolg ${ }^{28}$. Diese Strukturierung des Schuldverhältnisses wirkt sich auf die Beweislastverteilung aus ${ }^{29}$ : Bei einer Leistungshandlung ist der Gläubiger schlechter gestellt, denn

23 DEMOGUE, Traité des obligations en général, Bd. II, Effets des obligations, T. VI, Paris, 1932, n. 153 ff., n. 530 ff., 597 ff.

24 Vgl. Cass. civ., 20.5.1936, in Dalloz, 1936, I, S. 88; Cass. civ., 12.7.1960, in Dalloz, 1960, S. 101. Vgl. MONATERI, Cumulo di responsabilità contrattuale e extracontrattuale, Padova, 1989, S. 90 ff. ; MENGONI, Obbligazioni „di risultato“ e obbligazioni di „mezzi“, in Riv. dir. comm., 1954, I, S. 185, Fußn. 2.

25 H. MAZEAUD/L. MAZEAUD, Traité théorique et pratique de la responsabilité civile, 4. Aufl., Bd. I, Paris, 1947, Rn. 103 ff.; TUNC, Distinzione delle obbligazioni di risultato e delle obbligazioni di diligenza, in Nuova riv. dir. comm., 1947-48, I, S. 145. Ein kritischer Standpunkt wurde vertreten von CAPITANT, Les effets des obligations, in Rev. trim. dr. civ., 1932, S. 721 ff.; ESMEIN, Le fondement de la responsabilité contractuelle rapprochée de la responsabilité délictuelle, in Rev. trim. dr. civ., 1933, S. 627 ff.; Ders., Obligations, in Traité pratique de droit civil français hrsg. von Planiol und Ripert, Bd. VII, 2. Aufl., Paris, 1952, Rn. 378 ter, S. 498 f.; MARTON, Obligations de résultat et obligations de moyens, in Rev. trim. dr. civ., 1935, S. 499 ff.; RODIERE, La responsabilité civile, Paris, 1952, Rn. 1669, S. 288 ff.; COLIN/CAPITANT/JULLIOT DE LA MORANDIERE, Traité de droit civil, Bd. II, 2. Aufl., Paris, 1959, Rn. 691, S. 390 und Rn. 832, S. 464; CHABAS, Vers un changement de nature de l'obligation médical, in Juris class. pér., 1973, I, S. 2541.

26 Vgl. H. MAZEAUD, Essai de classification des obligations, a.a.O., S. 1 ff; DEMOGUE, a.a.O., T. VI, Rn. 597 ff., S. $642 \mathrm{ff}$.

27 VISINTINI, a.a.O., S. 202 ; Cass., 11.3.2002, n. 3492, in Giust. civ. Mass., 2002, S. 435.

28 BILANCETTI, La responsabilità penale e civile del medico, 5. Aufl., Padova, 2003, S. 950; Cass., 28.4.1994, in Riv. it. med. leg., 1997, S. 474. Dagegen, VISINTINI, a.a.O., S. 205.

29 Vgl. VINEY/JOURDAIN, Les conditions de la responsabilité, in Traité de droit civil hrsg. von Ghestin, 3. Aufl., Paris, 2006, S. 633 ff.; LE TOURNEAU, Responsabilité civile professionnelle, 2. Aufl., Paris, 2005, a.a.O.; CARBONNIER, Droit civil, Bd. IV, 22. Aufl., Paris, 2000, S. 288 ff.; SAVATIER, La théorie des obligations en droit privé economique, Paris, 1979, n. 128, S. 188 ff.; STARCK, Droit civil, Les Obligations, Paris, 1972, S. 525 ff., S. 611 ff. und S. 654 ff.; WEILL/TERRÉ, Droit civil, Les obligation, 4. Aufl., Paris, 1986, S. 399 ff. und S. 775 ff.; FROSSARD, La distinction des obligations de moyens et des obligations de résultat, Paris, 1965, S. 107 f.; DE 
er muss den positiven Beweis erbringen, dass der Schuldner die Sorgfaltspflicht verletzt hat, und somit dessen Verschulden nachweisen. Im Falle eines Leistungserfolgs ist der Erfolg in obligatione, und der Gläubiger muss nur unter Beweis stellen, dass dieser Erfolg nicht erreicht wurde, denn in diesem Fall wird das Verschulden des Schuldners vermutet. ${ }^{30}$

Der positive Anklang, den die Unterscheidung zwischen Leistungshandlung und Leistungserfolg gefunden hat, ist u.a. darauf zurückzuführen, dass sich in Frankreich langsam aber unaufhaltsam der Grundsatz des ,non-cumul“ zwischen vertraglicher und außervertraglicher Haftung durchgesetzt hat ${ }^{31}$. Unter ständigem Verweis auf - häufig interpolierte - Präzedenzfälle der Rechtsprechung ${ }^{32}$ hat die Lehre $^{33}$ in der Überzeugung, dass eine Verwechslung zwischen zwei streng getrennten Ebenen extrem gefährlich ist, das Kumulierungsverbot zu einem Grundsatz des positiven Rechts erhoben. Seine Anerkennung und die ständig steigende Mobilität der Grenzen ${ }^{34}$ zwischen den beiden Haftungsformen hat unvermeidbar dazu geführt, dass gewisse Verpflichtungen manchmal in den Vertrag einbezogen werden und der vertragliche Bereich dann aber wieder eingeschränkt wird. ${ }^{35}$

\section{Die Gleichstellung der vertraglichen und außervertraglichen Haftung im 20. Jahrhundert}

Im 19. Jahrhundert wurde davon ausgegangen, dass die Arzthaftung den in den Art. 1382 und 1383 fr. Cod. Civ. verankerten, allgemein geltenden Maßstäben der

LORENZI, voce Obbligazioni di mezzi e obbligazioni di risultato, in Dig. IV disc. priv., Bd. XII, Torino, 1995, S. 398.

30 Vgl. H. MAZEAUD, Essai de classification des obligations, a.a.O., S. 40 ff. ; TUNC, a.a.O., S. $130 \mathrm{ff}$. Vgl. auch CARBONE, Obbligazioni di mezzi e di risultato tra progetti e tatuaggi, in Corr. giur., 1997, S. 554; Cass., 7.2.1996, n. 973, in Corriere giur., 1996, S. 541 ff., mit Anm. von MARICONDA, Risarcimento del danno e onere della prova.

31 MONATERI, a.a.O.; GAMBARO, Responsabilità contrattuale ed extracontrattuale. Significato attuale di una distinzione tradizionale, Milano, 1993.

32 SAVATIER, Traité de la responsabilité civil en droit français, I, 2. Aufl., Paris, 1951, n. 148 f., S. 192 f.; D. CARUSI, Responsabilità del medico e obbligazione di mezzi, a.a.O., S. 506 ff.; MONATERI, Cumulo di responsabilità contrattuale e extracontrattuale, a.a.O.

33 Vgl. VINEY/JOURDAIN, a.a.O., S. 263 ff.; MAZEAUD/MAZEAUD/TUNC, Traité de la responsabilité civile, Bd. I, 6. Aufl., Paris, 1965, n. 173-207; MALAURIE/MALAURIE, Cours de droit civil, Les obligations, Paris, 1985, S. 370; WEILL/TERRÉ, Droit civil, 3. Aufl., Paris, 1985, n. 368; MARTY/RAYNAUD, Droit civil, Bd. II, Les obligations, Paris, 1962, n. 368; ESMEIN, Trois problèmes de responsabilité civile, in Rev. trim. dr. civ., 1934, S. 349 f.

34 BUSNELLI, Le mobili frontiere del danno ingiusto, in Contratto e impresa, 1985, S. 1 ff.

35 MONATERI, a.a.O., S. 107. 
Vorsicht und Sorgfalt unterlag. ${ }^{36}$ Ein Tendenzwandel erfolgte im Jahr 1936 durch die Cour de Cassation $^{37}$, als sich die vertragliche Natur des Arzt-PatientenVerhältnisses und der schuldrechtliche Inhalt der Leistung nicht als obligation déterminée de guérir le malade, sondern als obligation générale de prudence et diligence $^{38}$ oder besser gesagt als obligation de sécurité de moyens durchsetzte ${ }^{39}$. Derzeit folgt die Rechtsprechung nach wie vor der traditionellen Auffassung, obwohl nach Ansicht namhafter Autoren bei Fällen der Arzthaftung auf die strenge Unterscheidung zwischen den beiden Haftungsformen verzichtet werden sollte ${ }^{40}$.

Italien hat einen ähnlichen Weg wie Frankreich beschritten: Nach einer zunächst entschiedenen Befürwortung der Arzthaftung als außervertragliche Haftung $^{41}$ und einer lang anhaltenden Ungewissheit ${ }^{42}$ ist es heute im Wesentlichen unerheblich, auf welchen Titel der Kläger seinen Schadenersatzanspruch stützt ${ }^{43}$. Deshalb untersucht die Rechtsprechung - auch ultra petita - stets das Vorliegen

36 Vgl. AUBRY/RAU, Cours de droit civil français, 4. Aufl., Paris, 1871. Contra, MAZEAUD, Responsabilité délictuelle et responsabilité contractuelle, a.a.O., S. 612. Unter Anwendung der Bestimmungen über die Delikte und die Quasi-Delikte gemäß Art. 1151 f. des italienischen Codice civile von 1865 wurde die zivilrechtliche Haftung des Arztes auch von der italienischen Rechtsprechung in den Bereich des Schutzes aus unerlaubter Handlung eingereiht. Vgl. App. Roma, 8.6.1886, in Foro it., 1886, I, c. 714.

37 Cass. civ., 20.5.1936, in Dalloz, 1936, I, S. 88 mit Anm. von JOSSERAND.

38 Vgl. PLANCQUEEL, Obligations de moyens, obligations de résultat, in Rev. trim. dr. civ., 1972, S. 334.

39 Vgl. für alle, Trib. gr. ist. Meaux, 1.12.1961, in Gazette du Palais, 1962, II, S. 44. Vgl. TUNC, in Rev. trim. dr. civ., 1962, S. 635 f.; H.L. MAZEAUD, ivi, 1957, S. 524. LAMBERT-FAIVRE, Fondement et régime de l'obligation de sécurité, in Dalloz, Chr., 11, 1994, S. 84: „L'obligation de sécurité est par nature une obligation déterminée, l'objet de l'obligation étant la sécurité due en tout état de cause par l'auteur du dommage“.

40 VINEY/JOURDAIN, a.a.O., S. 290.

41 App. Milano, 18.4.1939, in Resp. civ. e prev., 1940, S. 120. Vgl. auch, Cass., 13.5.1931, in Ann. dir. comp., Bd. IX, III, S. 468, mit Anm. von MONTEL, In tema di responsabilità del medico e dell'ospedale verso l'ammalato per cura non riuscita; Cass., 27.7.1933, n. 2934, in Mass. Foro it., 1933, S. 566; Cass., 17.6.1936, n. 2107, in Foro it., 1936, I, S. 815. Vgl. GABBA, Anmerkung, in Foro it., 1899, I, S. 93.

42 Im Sinne der vertraglichen Haftung: Cass., 21.12.1978, n. 6141, in Foro it., 1979, I, S. 4; Cass., 1.3.1988, n. 2144, in Foro it., 1988, I, S. 2296, mit Anm. von PRINCIGALLI; Cass., 1.9.1999, n. 9198, in Giust. civ. Mass., 1999, S. 1877. Im Sinne der außervertraglichen Haftung: Cass., 24.3.1979, n. 1716, in Giur. it., 1981, I, 1, S. 297; Cass., 26.3.1990, n. 2428, in Giur. it., 1991, I, S. 600; Cass., 13.3.1998, n. 2750, in Foro it., 1998, I, S. 3521

43 Vgl. CASTRONOVO, L'obbligazione senza prestazione ai confini tra contratto e torto, in La nuova responsabilità civile, Milano, 1997, schon in Scritti in onore di Luigi Mengoni, Bd. I, Le ragioni del diritto, Milano, 1995, S. 147. 
Die Arzthaftung von gestern und das Medizinrecht von heute in rechtsvergleichender Perspektive

der beide Haftungsformen charakterisierenden Elemente ${ }^{44}$ und vertritt unter diesem Gesichtspunkt den Auffassungen des deutschen BGH ähnliche Standpunkte. ${ }^{45}$

Grundsätzlich standen die kontinentaleuropäischen Rechtssysteme ${ }^{46}$ also im letzten Jahrhundert dem Übergang von der vertraglichen auf die außervertragliche Haftung im Bereich des Medizinrechts ziemlich gleichgültig gegenüber. Diese Haltung ist auch heute noch überaus verbreitet, und es wurde eine Reihe von Grundsätzen ausgearbeitet, die unabhängig von der jeweiligen Haftungsform zur Anwendung kommen.

Obwohl im englischen Common Law der Klage gegen den Arzt traditionellerweise deliktische Natur beigemessen wird, gab es dort einen Zeitpunkt, zu dem bei der Klage auf assumpsit für das entgeltliche Arzt-Patienten-Verhältnis eine vertragliche Haftung zum Tragen $\mathrm{kam}^{47}$. In diesen Fällen war die consideration das zentrale Element: Konnte der Patient keine tangible consideration nachweisen, gingen die Gerichtshöfe davon aus, dass alleine der Umstand, dass sich der Patient der ärztlichen Behandlung unterzogen hatte, als sufficient consideration anzusehen war. ${ }^{48}$ Seit der Gründung des National Health Systems (NHS) wird einhellig der Standpunkt vertreten, dass zwischen Arzt und Patient kein Vertragsverhältnis besteht. ${ }^{49}$ Deshalb handelt es sich bei der typischen Haftungsklage gegenüber dem Arzt heute um eine action for negligence, während nur im Falle privater Behandlungen von einem Vertragsverhältnis ausgegangen wird.

44 Die Corte di Cassazione hat bereits ab dem Urteil Nr. 1282 von 1971 (in Giust. civ., 1971, I, S. 1417) die Behauptung aufgestellt, dass „Art. 2236 des Codice civile (...) nicht nur im vertraglichen, sondern auch im außervertraglichen Bereich anwendbar ist" („L'art. 2236 c.c. (...) è applicabile oltre che nel campo contrattuale anche in quello extracontrattuale"). Für eine Übersicht: PARTISANI, Il contratto atipico di spedalità e cura: nuove regole di responsabilità, in La Resp. civ., 2007, S. 1028. Der Grundsatz wurde incidenter tantum auch vom italienischen Verfassungsgerichtshof aufgestellt: C.Cost., 22.6.1970, n. 307, in Foro it., 1990, I, S. 2694.

45 BGH, 24.1.1995, in NJW, 1995, S. 1618; BGH, 24.1.1984, in NJW, 1984, S. 1403. Vgl. DEUTSCH/SPICKHOFF, Medizinrecht, 6. Aufl., Berlin u.a., 2008, S. 112 f., Rn. 165.

46 Für einen rechtsvergleichenden Überblick über die Arzthaftung vgl. die in Rev. int. dr. comp., 1976 veröffentlichte Übersicht mit den Beiträgen verschiedener namhafter Autoren: SAVATIER, La responsabilité médicale en France, S. 493 ff.; BIANCHI DESPINOSA/ZHARA BUDA, La responsabilité médicale en Italie, S. 531 ff.; PETITPIERRE, La responsabilité de droit privé du médicin: aperçu du droit suisse, $\mathrm{S}$. $567 \mathrm{ff}$.

47 Everard v. Hopkins (1615) 80 ER 1164 und Slater v. Baker and Stapleton (1767) 95 ER 860.

48 Coggs v. Bernard (1703) 92 ER 107.

49 Pfizer Corporation v. Ministry of Health (1965) AC 512 (HL). Anders in Canada: Pittman Estate v. Bain (1994) 112 DLR (4th) 257 (Ont. Gen. Div.). 


\section{Der Wandel der zivilrechtlichen Haftung in Europa von der Kodifikationszeit bis heute}

Für die weitere rechtsgeschichtliche Rekonstruktion ist im Übrigen festzuhalten, dass die zivilrechtliche Haftung selbst einen Wandel durchgemacht hat: An die Stelle der sanktionierenden Haftung des 19. Jahrhunderts, die auf einer auf den Schadensverursacher konzentrierten Schuldzuweisung aufgebaut war, trat im 20. Jahrhundert eine entschädigende Haftung, die auf das Risiko und auf das Schadensopfer konzentriert war. Das 21. Jahrhundert wird aller Wahrscheinlichkeit nach zum Jahrhundert der sogenannten ${ }^{50}$ responsabilité-anticipation, die auf die Prävention der wichtigsten Risiken und auf den Interessenschutz von Personengruppen ausgerichtet ist (mit dem Ziel ihrer Ausdehnung auf sämtliche Lebewesen und auch ihre Nachkommen).

\section{Die fundamentalen Änderungen der letzten Jahrzehnte}

In der Zeitgeschichte der Medizin sind in den letzten Jahrzehnten zwei fundamentale Änderungen eingetreten.

\section{a) Die technologische Revolution}

In erster Linie war eine regelrechte technologische Revolution zu beobachten: Die gesellschaftlichen Debatten, die Wissenschaft und die Medizin sind deshalb mit bis vor kurzer Zeit nicht vorhandenen Problemen konfrontiert. Meiner Auffassung nach geht es in diesem Zusammenhang um den Beginn und das Ende des Lebens: Die Geburt und den Tod, d.h. neben unerwünschten Geburten und Schwangerschaften um genetische Untersuchungen, künstliche Reproduktionstechniken, Frühgeburten und um die Aspekte der Kranken im Endstadium, die Euthanasie, das biologische Testament und den programmierten Selbstmord.

Diese Themen stehen in engem Zusammenhang mit der medizinischen und pharmakologischen Erprobung: Hoffnungslose Fälle und Grenzfälle legitimieren und stimulieren den Einsatz unkonventioneller Techniken, Medikamente oder Verfahren. Durch den technologischen und wissenschaftlichen Fortschritt sind der Bereich der Medizin, des Gesundheitswesens und der Pharmazeutik untrennbar zusammengewachsen: Bei einer Frühgeburt überschneidet sich der rein ärztliche Eingriff z.B. mit der Tätigkeit des Pflegepersonals, der medizinischen Hilfskräfte und der Krankenhausorganisation im weiten Sinne (vor allem im Hinblick auf die mechanischen Geräte, wie den Brutkasten), wobei der pharmakologische Beitrag nicht vergessen werden darf. Jeder Bereich kooperiert mit den anderen. Tritt ein Schadensereignis ein, könnte die Haftung bei jedem einzelnen oder bei sämtlichen Bereichen liegen.

Diese Entwicklung wird auch auf terminologischer Ebene deutlich: In Italien ist z.B. die „responsabilità del medico“ zur „responsabilità medica“ geworden,

50 THIBIERGE, Avenir de la responsabilité, responsabilité de l'avenir, in Recueil Dalloz, 2004, Nr. 9, S. 581. 
was also bedeutet, dass sich die Haftung auch auf die Einrichtung des Gesundheitswesens erstreckt; in England wurde die „medical negligence " zur „,clinical negligence ${ }^{\text {“51. }}$.

Ausweislich der rechtsvergleichenden Gegenüberstellung ist dieser Ansatz sowohl im Civil law als auch im Common law bereits seit längerer Zeit zum Ausdruck gekommen: In den Vereinigten Staaten wird die Materie als Health Law bezeichnet $^{52}$, in England spricht man dagegen von Medical $L_{a w}{ }^{53}$, in Deutschland von Medizinrecht (und dies ist Erwin Deutsch zu verdanken) ${ }^{54}$. In diesen Ländern reicht die Materie von der informierten Zustimmung des Patienten über die Haftung für die eigentliche ärztlichen Behandlung und für den allgemeinen Gesundheitsdienst des Krankenhauses bis zum Schutz der personenbezogen Daten und umfasst schließlich die Erprobung und das Inverkehrbringen von Medikamenten sowie die Produkthaftung.

In den Rechtssystemen romanistischer Tradition, wie Frankreich und Italien, wird die Materie dagegen noch nicht als eigenständiger und charakteristischer Rechtsbereich angesehen. Wahrscheinlich konnte sich das Medizinrecht aufgrund der strengen Unterscheidung zwischen Leistungshandlung und Leistungserfolg bisher nicht von der Regelung der beruflichen Haftung loslösen, die der Arzt mit anderen Berufskategorien gemein hat. Denn trotz der behaupteten Überwindung der klassischen Unterscheidung ${ }^{55}$ wird sie von Lehre und Rechtsprechung laufend verwendet.

\section{b) Die exponentielle Aufmerksamkeit für die Person}

Der zweite Wandel ist eine Folge der exponentiellen Steigerung der Aufmerksamkeit für die Person und für die hohe Wertschätzung der mit der Persönlichkeit verbundenen Aspekte. Vor allem in den Rechtssystemen, die der Verfassung groBe Bedeutung beimessen, setzen sich zivilrechtliche Freiheiten, das Persönlichkeitsrecht und insbesondere der Grundsatz der Selbstbestimmung der Person energisch durch. ${ }^{56}$ Aus einer rechtsvergleichenden Gegenüberstellung ergibt sich, dass die Informationspflicht in diesen Ländern im Bereich des Medizinrechts eine fundamentale Rolle spielt, während sich die Situation in den Ländern, in denen die Verfassung eine untergeordnete Bedeutung einnimmt oder überhaupt nicht vorhanden ist, völlig anders gestaltet. Man denke zum Beispiel an einige Systeme, die ein und dieselbe Tradition des Common Law gemein haben: Die Vereinigten Staaten und Kanada, wo die Verfassung den Kernpunkt des gesamten Rechtssystems darstellt, und England, wo es keine Verfassung gibt: In den Vereinigten Staaten

51 GRUBB, Duties in Contract and Tort, in GRUBB (Hrsg.), Principles of Medical Law, 2. Aufl., Oxford, 2004, S. 313.

52 AMERICAN HEALTH LAWYERS ASSOCIATION, Fondamentals of Health Law, 4. Aufl., Washington, 2008.

53 GRUBB (Hgb.), Principles of Medical Law, 2. Aufl., Oxford, 2004.

54 DEUTSCH/SPICKHOFF, a.a.O.

55 Cass., 13.4.2007, n. 8826, in www.cortedicassazione.it.

56 Von BAR, Der Einfluß des Verfassungsrechts auf die westeuropäischen Deliktsrechte, in RabelsZ, 59 (1995), S. 214 ff. 
und in Kanada muss der patient's consent ,informed“" $\operatorname{sein}^{57}$, in England nur ,, re$a l^{\text {‘58}}$. In Kanada geht der Supreme Court davon aus, dass die Zustimmung im Falle von fraud oder misrepresentation ungültig ist, auch wenn sie ,real ${ }^{659}$ ist, während die englischen Gerichtshöfe den gegenteiligen Standpunkt vertreten ${ }^{60}$. In Kanada stützt sich die Würdigung der duty of information auf den ,reasonable patient test ${ }^{\star 61}$, in England dagegen auf den sog. „Bolam test ${ }^{\star 62}$, dessen Grundlage doctor's reasonable behavior darstellt ${ }^{63}$.

\section{Die „responsabilité-anticipation“ und die heutigen verschiedenen Lösungen der europäischen Rechtsordnungen}

Die steigende Aufmerksamkeit für das menschliche Wesen, die ständig wachsende Effizienz der Rechtsmittel für dessen Schutz und die technologische Revolution haben zu einer spontanen Differenzierung von medizinischen Sachverhalten geführt, die heute meiner Meinung nach in zwei Gruppen unterteilt werden können:

1. die unheilbaren oder unbekannten Fälle bzw. die Notfälle;

2. alle anderen Fälle, d.h. die heilbaren Fälle, die die wesentlichen Quellen der Rechtsprechung zum Thema Arzthaftung darstellen.

Bei der ersten Gruppe sind die Fehlerrisiken höher und auf rechtlicher Ebene geht die Tendenz dahin, den Arzt von der Haftung freizustellen, ihn in seinem Einsatz nicht zu entmutigen. Bei der zweiten Gruppe neigt man dagegen zu einem gesteigerten Patientenschutz, vor allem durch den Einsatz von Techniken der ,vorweggenommenen Haftung“ (die bereits erwähnte responsabilité anticipation), die in den jeweiligen Rechtssystemen entweder ausdrücklich vorgegeben sein (in von der Rechtsprechung oder vom Gesetzgeber aufgestellten Grundsätzen) oder in nicht wörtlich festgehaltener Formen (Kryptotypen) aufscheinen können. ${ }^{64}$

57 Canterbury v. Spence (1972) 464 F 2d 772 (DC Cir.).

58 Ciarlariello v. Schacter (1993) 100 DLR (4th) 609.

59 Reibl v. Hughes (1980) 114 DLR (3d) 1, 11; PICARD/ROBERTSON, Legal Liability of Doctors and Hospital in Canada, 3. Aufl., Scarborough, 1996, S. 57 ff.

60 Chatterton v. Gerson (1981) QB 265; Appleton v. Garrett (1997) 8 Med LR 77.

61 Reibl v. Hughes, a.a.O.

62 Bolam v. Friern Hospital Management Comittee (1957) 2 All ER 118; JONES, Medical Negligence, 3. Aufl., Andover, 2003.

63 Diesem Grundsatz folgte das House of Lords in den Urteilen Whitehouse v. Jordan (1981) 1 All ER 267; Bolitho v. City and Hackney HA (1997) 4 All ER 771.

64 Zum Begriff der Kryptotypen vgl. SACCO, La comparazione giuridica, in SACCO/GAMBARO, Sistemi giuridici comparati, in Trattato di diritto comparato unter der Leitung von Sacco, 2. Aufl., Torino, 2002, S. 7 ff. 
Die Arzthaftung von gestern und das Medizinrecht von heute in rechtsvergleichender Perspektive

\section{Der Ermessensspielraum der niederländischen Richter}

In Holland wurde die responsabilité anticipation kodifiziert ${ }^{65}$, indem in die Regelungen über die zivilrechtliche Haftung im Nieuw Burgerlijk Wetboek von 1992 Maßstäbe für die Festsetzung des Schadens aufgenommen wurden, die die Art der Haftung, das zwischen den Parteien bestehende Rechtsverhältnis und deren jeweilige Leistungsfähigkeit ebenso berücksichtigen wie die Frage, ob ein Versicherungsschutz besteht oder nicht ${ }^{66}$.

\section{Die überwiegende Erheblichkeit der Aufklärungspflicht in Frankreich, Deutschland, Italien, Österreich und in der Schweiz}

In Frankreich, Italien, Deutschland, Österreich und in der Schweiz wurde die responsabilité anticipation im Rahmen einer langen, aber unaufhaltsamen Forschung nach Lösungen zugunsten des Patienten durch die Rechtsprechung umgesetzt. In diesem Zusammenhang konzentrierte sich die Aufmerksamkeit auf die Nichterfüllung der Informationspflicht: Mit der Begründung, dass es für den Patienten schwierig ist, einen negativen Beweis für den Informationsmangel zu erbringen, bzw. dass der Beweis für den Arzt näherliegend ist als für den Patienten, hat die Rechtsprechung eine Beweislastumkehr vorgenommen. ${ }^{67}$ In diesen Ländern war in den letzten Jahren eine erhebliche Steigerung der auf die Verletzung der Informationspflicht gestützten Prozesse zu beobachten. ${ }^{68}$ Diese causa petendi

65 HARTKAMP, Das neue niederländische Bürgerliche Gesetzbuch aus europäischer Sicht, in RabelsZ, 57 (1993), S. 672 und S. 679 f.

66 Art. 6.109, Abs. 1 und 2, NBW: ,(1) Indien toekenning van volledige schadevergoeding in de gegeven omstandigheden waaronder de aard van de aansprakelijkheid, de tussen partijen bestaande rechtsverhouding en hun beider draagkracht, tot kennelijk onaanvaardbare gevolgen zou leiden, kan de rechter een wettelijke verplichting tot schadevergoeding matigen. (2) De matiging mag niet geschieden tot een lager bedrag dan waarvoor de schuldenaar zijn aansprakelijkheid door verzekering heeft gedekt of verplicht was te dekken. (...)“. Übersetzung ins Deutsche von NIEPER, Buch 6, Allgemeiner Teil des Schuldrechts, in NIEPER/WESTERDIJK, Niederländisches Bürgerliches Gesetzbuch, München, 1995.

67 Vgl. DEUTSCH, Neues zur ärztlichen Aufklärung im Ausland, in MedR, 2005, S. 464 ff.

68 Frankreich: CASTELLETTA, a.a.O., S. 16, Rn. 12.11; Italien: FERRANDO, Libertà, responsabilità e procreazione, Padova, 1999; FRANZONI, La responsabilità del medico fra diagnosi, terapia e dovere di informazione, in La responsabilità civile, 2005, S. 584 ff.; Deutschland: SPICKHOFF, Die Entwicklung des Arztrechts 2004/2005, in NJW, 2005, S. 1698; DEUTSCH/SPICKHOFF, a.a.O., S. 164, Rn. 245; Österreich: HÖFTBERGER, Österreichische Rechtsprechung zur Arzthaftung, in MedR, 2000, S. 509 ff.; HARRER, in KOPETZKI/ZAHRL (Hrsg.), Behandlungsanspruch und 
wird auch bei Arztfehlern bevorzugt eingesetzt, für die der Patient sonst den positiven Beweis erbringen müsste. ${ }^{69}$ Im Jahr 1997 hat die Rechtsprechung ihren Standpunkt sowohl in Frankreich ${ }^{70}$ als auch in Italien ${ }^{71}$ radikal geändert und behauptet, dass derjenige, dem eine besondere Informationspflicht obliegt, den Beweis für die Erfüllung dieser Pflicht erbringen muss. In Frankreich haftet der Arzt im Übrigen bei Verletzung der Informationspflicht vollumfänglich für den verursachten Schaden, wenn er die Erfüllung seiner Informationspflicht nicht unter Beweis stellt. ${ }^{72}$ Der Grundsatz wurde darüber hinaus auch auf gesetzlicher Ebene verankert. ${ }^{73}$ In Deutschland erkennt die Rechtsprechung auf vollen Schadenser-

Wirtschaftlichkeitsgebot, Wien, 1998, S. 49; Schweiz: KUHN, Die Arzthaftung in der Schweiz, in MedR, 1999, S. $250 \mathrm{ff}$.

69 QUAAS/ZUCK, Medizinrecht, München, 2005, § 13, Rn. 69, sprechen von einem „Auffangtatbestand“.

70 Cass. civ., 25.2.1997, in Dalloz 1997, IR, S. 81. Für die früher vertretene Auffassung s. Cass. Civ., 29.5.1951, in Bull. Civ., n. 162 und in Dalloz, 1952, Jur., S. 53, mit Anm. von SAVATIER; Cass. civ., 4.4.1995, in Bull. civ., I, n. 159.

71 Cass., 24.9.1997, n. 9374, in Mass. Foro it, 1997.

72 Cass. Civ., 11.2.1986, unveröffentlicht, zitiert von CASTELLETTA, Responsabilité médical. Droits des malades, 2. Aufl., Parigi, 2004, S. 71.

73 Art. L. 1111-2 Code de la santé publique, verändert durch Loi 2005-370 vom 22.4.2005: „(1) Toute personne a le droit d'être informée sur son état de santé. Cette information porte sur les différentes investigations, traitements ou actions de prévention qui sont proposés, leur utilité, leur urgence éventuelle, leurs conséquences, les risques fréquents ou graves normalement prévisibles qu'ils comportent ainsi que sur les autres solutions possibles et sur les conséquences prévisibles en cas de refus. Lorsque, postérieurement à l'exécution des investigations, traitements ou actions de prévention, des risques nouveaux sont identifiés, la personne concernée doit en être informée, sauf en cas d'impossibilité de la retrouver. (2) Cette information incombe à tout professionnel de santé dans le cadre de ses compétences et dans le respect des règles professionnelles qui lui sont applicables. Seules l'urgence ou l'impossibilité d'informer peuvent l'en dispenser. (3) Cette information est délivrée au cours d'un entretien individuel. (4) La volonté d'une personne d'être tenue dans l'ignorance d'un diagnostic ou d'un pronostic doit être respectée, sauf lorsque des tiers sont exposés à un risque de transmission. (5) Les droits des mineurs ou des majeurs sous tutelle mentionnés au présent article sont exercés, selon les cas, par les titulaires de l'autorité parentale ou par le tuteur. Ceux-ci reçoivent l'information prévue par le présent article, sous réserve des dispositions de l'article L 1111-5. Les intéressés ont le droit de recevoir eux-mêmes une information et de participer à la prise de décision les concernant, d'une manière adaptée soit à leur degré de maturité s'agissant des mineurs, soit à leurs facultés de discernement s'agissant des majeurs sous tutelle. (6) Des recommandations de bonnes pratiques sur la délivrance de l'information sont établies par la Haute Autorité de santé et homologuées par arrêté du ministre chargé de la santé. (7) En cas de litige, il appartient au professionnel ou à l'établissement de santé d'apporter la preuve que l'information a été délivrée à l'intéressé dans les conditions prévues au présent article. Cette preuve peut être apportée par tout moyen.”. 
Die Arzthaftung von gestern und das Medizinrecht von heute in rechtsvergleichender Perspektive

satz, wenn der Patient einen ,echten Entscheidungskonflikt" hinsichtlich der Aufklärung substantiiert darlegt, ohne diesen beweisen zu müssen ${ }^{74}$.

In Italien ist die Rechtsprechung sogar noch weiter gegangen und hat die Beweislastumkehr auf sämtliche Fälle erweitert, in denen eine Nichterfüllung der Verpflichtung vorliegt: Die Vereinigten Senate der Corte di Cassazione ${ }^{75}$ haben angemerkt, dass „,der Gläubiger den Titel der Verpflichtung beweisen und nur die Nichterfüllung darlegen muss, während der Schuldner die rechtserlöschende Tatsache unter Beweis stellen oder den haftungsbefreienden Beweis nach Art. 1218 it. Cod. Civ. erbringen muss" ${ }^{\text {"7 }}$.

Demzufolge wurde vor allem in Frankreich und Deutschland eine Verteidigungsstrategie durch die Einführung von Risk Management-Prozeduren und die Ausarbeitung strenger Protokolle entwickelt: Der Patient wird überaus gewissenhaft informiert und erhält stets zahlreiche Formulare und detaillierte Informationsbroschüren. Bei bestimmten Eingriffen (wie bei Schönheitsoperationen, bei denen meistens das Ergebnis gewährleistet wird ${ }^{77}$ ) wird ein Vertrag abgeschlossen, in dem jede Phase der Operation, die erwarteten Mindestergebnisse, die möglichen Risiken usw. genau beschrieben sind.

74 BGH, 1.2.2005, in NJW, 2005, S. 1364. Vgl. auch DEUTSCH, Neues zur ärztlichen Aufklärung im Ausland, a.a.O., S. 465; SPICKHOFF, Die Entwicklung des Arztrechts 2006/2007, in NJW, 2007, S. 1632.

75 Cass., Sez. Un., 30.10.2001, n. 13533, in Foro it., 2002, I, S. 769, mit Anm. von LAGHEZZA, Inadempimenti ed onere della prova: le sezioni unite e la difficile arte del rammendo; in Contr., 2002, S. 113, mit Anm. von CARNEVALI, Inadempimento e onere della prova; in Nuova Giur. civ. comm., 2002, I, S. 349, mit Anm. von MEOLI, Risoluzione per inadempimento ed onere della prova.

76 „Il creditore deve dimostrare il titolo dell'obbligazione ed allegare soltanto l'inadempimento, mentre il debitore deve dedurre il fatto estintivo o la prova liberatoria dell'art. 1218 c.c.".

77 In Frankreich wird die Leistungshandlung des Schönheitschirurgen überaus streng bewertet (CA Paris, 16.6.1995, in Dalloz, 1995, IR, S. 194) und die Informationspflicht ist „total“ (Cass. civ., 14.1.1992, n. 90-10.870, in Bull. civ., I, n. 16). Für Deutschland: DEUTSCH/GEIGER, Medizinischer Behandlungsvertrag, in Gutachten und Vorschläge zur Überarbeitung des Schuldrechts, 1982, S. 1095 f.; für Italien: Cass., 8.8.1985, n. 4394, in Foro it., 1986, I, S. 121 f., mit Anm. von PRINCIGALLI; VACCÀ, L'intervento di chirurgia estetica è obbligazione di risultato?, in Resp. civ. e prev., 1986, S. 44; ROMANO, Considerazioni in tema di responsabilità contrattuale del medico per violazione del dovere di informazione, in Giur. it., 1987, I, 1, S. 1136; COSTANZA, Informazione del paziente e responsabilità del medico, in Giust. civ., 1986, I, S. 1432; Trib. Trieste, 14.4.1994, in Resp. civ. e prev., 1994, S. 768, mit Anm. von F. und C. PONTONIO, La responsabilità del chirurgo estetico: obbligazione di mezzi o di risultato?; PERULLI, a.a.O., S. $450 \mathrm{f}$. 


\section{Die policy des englischen House of Lords}

In England übernimmt die duty of information dagegen wie gesagt eine stark untergeordnete Bedeutung, denn der Informationsmangel bewirkt einen Zustimmungsmangel, und der Arzt wird auf der Grundlage der tort of battery zur Verantwortung gezogen. Im Rahmen der Arzthaftung ist die englische Rechtsprechung bei der Anwendung der tort of battery jedoch äußerst zurückhaltend. ${ }^{78}$ Alle gegen Ärzte erhobenen Schadenersatzklagen wegen breach of duty sind actions of negligence. ${ }^{79}$ Die Beweislast obliegt ausschließlich dem claimant, d.h. dem Patienten, obwohl eine Umkehr der Beweislast versucht ${ }^{80}$, vom House of Lords aber energisch abgewiesen wurde ${ }^{81}$. Der klassische ,but for test" für den Beweis des kausalen Zusammenhangs ist jedoch zu unflexibel, und deshalb hat das House of Lords in den Fällen der Arzthaftung das Kriterium der „material contribution to the injury" eingeführt ${ }^{82}$ : Die Haftung des Arztes liegt dann vor, wenn sein Verhalten materiell zum Entstehen des Schadens beigetragen hat. Diese neue Ansicht entsprang der vom Gerichtshof gezeigten Bereitschaft, das Verschulden von einigen den Kläger de facto begünstigenden Elementen abzuleiten. Die Prüfung erfolgt durch äußerst technische gesetzliche Analysen, in denen zum Zweck der Ermittlung des kausalen Zusammenhangs komplexe linguistische Formeln auf die Tatsachen angewendet werden.

\section{Anregungen aus den skandinavischen und indischen Medizinrechtssystemen}

Abschließend kann behauptet werden, dass sich die Rechtsprechung grundsätzlich auf die eine oder andere Weise darum bemüht, den objektiven Konflikt zwischen der Notwendigkeit des förmlichen Nachweises der Arzthaftung und dem Wunsch nach Gerechtigkeit gegenüber dem Patienten zu lösen. Diese Entscheidung ist ein reines Problem der policy bzw. der Rechtspolitik. Im Vergleich zu allen anderen Fällen der zivilrechtlichen Haftung ist diese Frage jedoch im Bereich des Medizinrechts überaus heikel, denn einerseits gestalten sich die Tatsachenelemente aufgrund des Vorliegens zahlreicher variabler Werte besonders komplex, und andererseits geht es um das Leben und die Gesundheit eines Menschen. Die primären und auf nationaler Ebene anerkannten Zielsetzungen sind auf den Patientenschutz

78 Hills v. Potter (1983) 3 All ER 716, 728; Chatterton v. Gerson (1981) QB 432, 443; Abbass v. Kenney (1995) 31 BMLR 157, 163; The Creuzfeldt-Jakob Disease Litigation (1995) 54 BMLR 1.

79 GRUBB, Duties in Contract and Tort, a.a.O., S. 316, Rn. 5.06; Pfizer Corporation v. Ministry of Health (1965) AC 512 (HL).

80 McGhee v. National Coal Board (1973) 1 WLR 1.

81 Wilsher v. Essex AHA (1986) 3 All ER 801.

82 Bomington Castings v. Warlow (1956) AC 613; Holtby v. Brigham \& Cowan (Hill) Ltd (2000) 3 All ER 421 (CA). 
und die Effizienz des Systems gerichtet, und diese Aufgabe ist oft der Quadratur des Kreises gleichzustellen. Ein derart hochgestecktes Ziel kann in diesem Sinne nur durch ein System erreicht werden, in dem die Schäden des Patienten auf der Grundlage der jeweiligen Bedürfnisse und nicht auf der Grundlage des Verschuldensnachweises ersetzt werden.

Dieser Weg wurde von den skandinavischen Ländern beschritten, wo die Regeln der zivilrechtlichen Haftung de facto durch die Schaffung eines öffentlichen Versicherungssystems ersetzt wurden ${ }^{83}$ : Nimmt der Schaden gewisse Ausmaße an, wird dem geschädigten Patienten ein Pauschalersatz zuerkannt, der selbstverständlich unter der Summe liegt, die er im Falle des Obsiegens in einem Prozess in anderen europäischen Ländern erhalten würde, aber keine Kosten für Patienten und Schädiger verursacht. ${ }^{84}$ Prozedural gesehen ist dieser Mechanismus im Übrigen sehr einfach, denn der Patient muss lediglich den Beweis des Kausalzusammenhangs zwischen dem Schadensereignis und dem medizinischen, sanitären oder pharmazeutischen Vorfall erbringen. ${ }^{85}$

In Zukunft werden meiner Meinung nach zwei Phänomene mit sozialer Bedeutung eine fundamentale Rolle spielen und voraussichtlich einen direkten Einfluss auf das System der Arzthaftung ausüben:

- die geänderte Einstellung der Gesellschaft gegenüber dem professionista, der nicht mehr als Verwahrer des absoluten Wissens, sondern als Dienstleister angesehen wird;

- die geänderte Einstellung des Rechts gegenüber den Adressaten der Regeln, und hier beziehe ich mich insbesondere auf die Aufmerksamkeit, die die nationalen Gesetzgeber und der Gemeinschaftsgesetzgeber in den letzten Jahrzehnten dem Verbraucher und seinem Schutz gewidmet haben.

In diesem Sinne ist der Patient ein Verbraucher von Gütern (Medikamente und Maschinen) und Dienstleistungen (Tätigkeit des Arztes und der medizinischen Hilfskräfte, Krankenhaus), der angesichts des Umstandes, dass dieser Verbrauch seiner Gesundheit dient, eines besonderen Schutzes bedarf. ${ }^{86}$ Im Übrigen ist nicht von der Hand zu weisen, dass der Patient als typischer Verbraucher eine unterlegene Stellung einnimmt, und zwar auf drei Ebenen, d.h. informativ, psychologisch und wirtschaftlich.

In diesem Zusammenhang ist Indien ein vorbildhaftes Beispiel. Die Entwicklung, die das indische Rechtssystem dazu bewegt hat, die Verbrauchergerichte auch für Rechtsstreitigkeiten in Sachen ärztlicher, sanitärer und pharmazeutischer

83 LILIE/FISCHER, Ärztliche Verantwortung im europäischen Rechtsvergleich, in Schriftenreihe der Juristischen Fakultät Halle, Bd. 7, Köln u.a., 1999, S. 156.

84 LILIE/FISCHER, a.a.O., S. $157 \mathrm{f}$.

85 LILIE/FISCHER, a.a.O., S. 160.

86 HALL/SCHNEIDER, Patients as Consumers: Courts, Contracts, and the new Medical Marketplace, in Michigan Law Review, Bd. 106, N. 4, 2008, S. 643 ff. 
Haftung zugänglich zu machen, ist zweifellos überaus beeindruckend und äußerst interessant. ${ }^{87}$ In Indien sind diese drei Bereiche des Medizinrechts im Übrigen stark voneinander abhängig, denn das System ist angesichts der Koexistenz der herkömmlichen Heilkunde (die in vier Schulen gegliedert ist: Ayurveda, Siddha, Unani und Amtchi) und der westlichen Medizin, einschließlich Allopathie und Chirurgie, besonders komplex. ${ }^{88}$

Im Vergleich zur Lösung der skandinavischen Länder wurde die Effizienz des Systems auch durch die alternative indische Lösung, d.h. die Erweiterung der Zuständigkeit der Verbrauchergerichte auf die geschädigten Patienten, gesteigert und der Patient besser geschützt, vor allem deshalb, weil der Verbraucher insbesondere auf prozessualer Ebene in den Genuss einer Reihe von Erleichterungen, wie der Beweislastumkehr, der Vereinfachung des Prozesses und dessen informellen Verlaufs sowie der favor consumatoris ${ }^{89}$, kommt.

87 DAVID ANNOUSSAMY, India, in A. DIURNI (Hrsg.), Percorsi mondiali di diritto privato e comparato, Milano, 2008, S. 198.

88 DAVID ANNOUSSAMY, a.a.O., S. $200 \mathrm{ff}$

89 DAVID ANNOUSSAMY, a.a.O., S. $209 \mathrm{ff}$ 